DOI:10.2478/rrlm-2019-0039

\title{
The evaluation of the reverse algorithm for syphilis screening in blood donors
}

\author{
Rukiye Berkem ${ }^{1 *}$, Ayşe Esra Karakoç ${ }^{1}$ \\ 1. Ankara Education and Research Hospital, Turkey
}

\begin{abstract}
Background: In Turkey, prior to transfusion and apheresis, it is mandatory to screen blood for HBsAg, anti-HCV, anti-HIV 1/2, and syphilis. In recent years, efforts have been made to create effective diagnostic algorithms for screening, and as a screening strategy, many countries have switched from traditional algorithms to reverse algorithms. This study was carried out to evaluate the results we obtained after changing to chemiluminescence immunoassay (CLIA) based reverse algorithm, which is more sensitive and specific than the traditional algorithm and VDRL test we currently use for syphilis screening. Methods: The screening algorithm was initiated with the treponemal CLIA method. The reactive samples were tested using a nontreponemal test VDRL and a second treponemal test TPHA. In the descriptive analyses, the averages, numbers, and percentage distributions were calculated. The group comparisons were undertaken with the chi-square test, accepting $p<0.05$ as statistically significant. Results: Of the 5,053 samples that were tested with CLIA syphilis TPA assay 5,043 were negative (99.802\%) and 10 were positive reactive $(0.198 \%)$, and the difference between the two was statistically significant $(p<0.001)$. Ten samples that were recurrently reactive were analyzed by VDRL and TPHA tests simultaneously. VDRL was negative in all 10 samples, whereas TPHA was negative in one sample, but positive in nine samples at a titer of $\geq 1 / 160$. Conclusion: Based on the results of this study, we decided to use the CLIA method and reverse algorithm instead of using the traditional algorithm and VDRL as the initial screening method.
\end{abstract}

Keywords: blood donors, syphilis screening tests, confirmation, reverse algorithm

Received: 11 th April 2019; Accepted: 30 ${ }^{\text {th }}$ September 2019; Published: $12^{\text {th }}$ October 2019

\section{Introduction}

The World Health Organization (WHO) recommends the use of HBsAg, anti-HCV, anti-HIV $1 / 2$, and syphilis tests as pre-transfusion infection screening tests prior to blood and apheresis donations in all countries $(1,2)$. In Turkey, these screening tests are mandatory for blood donors.
WHO reported that there are 12 million new syphilis cases worldwide every year and recommends that donor screening should be undertaken with extremely sensitive methods to reduce the risk of syphilis transmission by transfusion $(1,2)$.

For the screening of syphilis (Treponema pallidum), methods that can detect the presence of

*Corresponding author: Rukiye Berkem, University of Health Sciences, Ankara Training and Research Hospital, Department of Medical Microbiology, Ankara, 06230, Turkey. E-mail: rukiyeberkem@yahoo.com 
nonspecific antibodies or specific treponemal antibodies are used. The techniques commonly used for syphilis screening include the Venereal Disease Research Laboratory (VDRL) test that employs a nonspecific antigen containing lecithin and cardiolipin; the highly sensitive and specific $T$. pallidum hemagglutination (TPHA) test involving the use of rapid plasma reagin (RPR) and/or treponemal antibodies; the T. pallidum particle agglutination test (TPPA); and the enzyme immunoassay (EIA) test (1, 3-6).

Recent studies on transfusion medicine focused on the creation of effective diagnostic algorithms for the screening of blood donors $(1,4,5)$. This study was conducted to evaluate the results of the chemiluminescence immunoassay (CLIA) method based on a reverse algorithm, which is more sensitive and specific than currently used traditional algorithm and VDRL test for syphilis screening.

\section{Materials and methods}

This study had a prospective design. The volunteer blood donors that applied to the blood center over a year were informed by the "Informed Consent Form" and they provided their written consent. Subsequently, they were asked to complete the "Blood Donor Registration Form" and the "Blood Donation Form". Medical evaluations including the donors' risk/behavior assessment were performed. Blood samples were taken for microbiological screening tests from the volunteers who were found to be suitable for blood donation, without exhibiting risky behavior or signs of infection. The prospective donors were tested for hepatitis B, hepatitis C, human immunodeficiency virus (HIV), and syphilis infections, which are mandatory for the screening of all blood and blood components in Turkey. Syphilis screening was performed using the Immunodiagnostic Products Syphilis TPA Assay (Ortho-Clinical Diagnostics, Vitros 3600) based on the CLIA method, which qualitatively investigates the $\operatorname{IgG}$ and $\operatorname{IgM}$ antibodies that form against $T$. pallidum-specific antigens (sensitivity 100\%, specificity 99.98\%), TPHA (Plasmatec microhemagglutination, UK; specificity 99.5\%, sensitivity 100\%), and VDRL (sensitivity $88.5 \%$, specificity $98.9 \%$ ) (Immutrep VDRL Antigen, Omega Diagnostics, UK). The tests were conducted and interpreted according to the manufacturer's recommendations. The results of the syphilis TPA assay were interpreted based on the $\mathrm{S} / \mathrm{Co}$ values (Table 1 ).

The reverse algorithm was applied as the syphilis screening strategy: First, the syphilis TPA assay was run; then, tittered VDRL and TPHA tests were simultaneously performed on the recurrent reactive samples (Figure 1).

For statistical evaluation, descriptive data, including the averages, numbers and percentage distributions were calculated. The chi-square

Table 1. Interpretation of the syphilis TPA assay based on the $S / C o$ values

\begin{tabular}{lc}
\hline VITROS Syphilis & \\
TPA Test Result $(\mathbf{S} / \mathbf{C o})$ & \\
\hline$<0.80$ & Negative \\
\hline$\geq 0.80$ to $<1.20$ & Borderline \\
\hline$\geq 1.20$ & Reactive \\
\hline$S$
\end{tabular}

S/Co: Signal/Cut Off

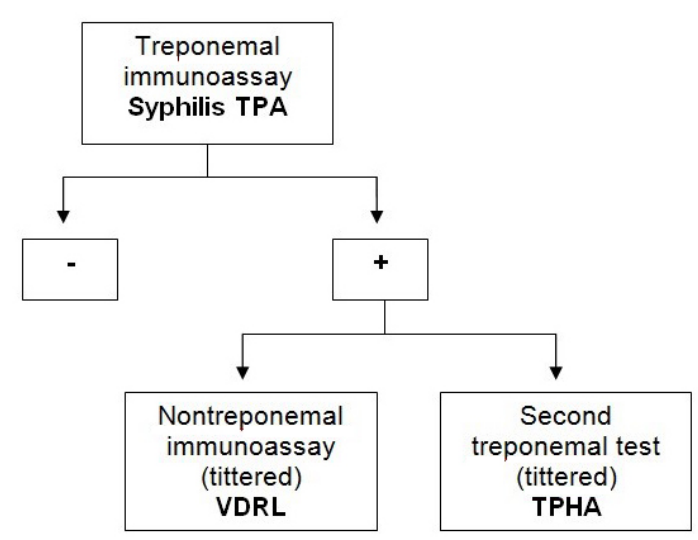

Fig. 1. Reverse syphilis screening algorithm 
test was applied for the group comparisons, and $\mathrm{p}<0.05$ was considered statistically significant.

\section{Results}

Of the 5,053 blood donors aged 18 to 65 years, 4,951 were male $(98 \%)$ and $102(2 \%)$ were female. The serum samples were first tested using the CLIA syphilis TPA assay, which revealed that 5,043 samples $(99.802 \%)$ were negative and 10 samples $(0.198 \%)$ were reactive, with a statistically significant difference between the two $(\mathrm{p}<$ 0.001 ). For the 10 samples found to be recurrent reactive, the VDRL and TPHA tests were performed simultaneously. All these 10 samples had VDRL negativity, whereas according to TPHA, only one sample was negative, and nine samples were positive at a titer of $\geq 1 / 160$. TPHA was repeated on the negative sample and the result was the same. Table 2 presents the results of the $\mathrm{S} / \mathrm{Co}$ ratios of the CLIA syphilis TPA assay, and the results of the VDRL and TPHA tests.

Of the blood donors with reactive CLIA syphilis TPA assay results, nine were male and one was female, with the mean age of 41.5 years, ranging from 28 to 52 years. Blood products that were found positive in any of the three tests were not used for donation and were destroyed.

Attempts were made to inform the ten blood donors with syphilis positivity about the results of the tests, and three of them returned to the blood center for the collection of a new blood sample. The remaining seven donors could not be reached due to inaccurate contact information. The blood samples of the three individuals were retested one month after the first evaluation, and the TPHA titers were observed to double, but the VDRL test was still negative (Table 3). The three blood donors with repeated samples and test results were referred to the infectious diseas-

Table 2. The results of the syphilis TPA assay, VDRL and TPHA tests

\begin{tabular}{lcccc}
\hline $\begin{array}{l}\text { Sample } \\
\text { Number }\end{array}$ & $\begin{array}{c}\text { VITROS Syphilis TPA S/Co } \\
\text { Reactive }\end{array}$ & VDRL & \multicolumn{2}{c}{ TPHA } \\
\hline 1 & 87.4 & Negative & $1 / 160$ & Positive \\
\hline 2 & 216 & Negative & $1 / 320$ & Positive \\
\hline 3 & 4.08 & Negative & Negative \\
\hline 4 & 86.6 & Negative & $1 / 320$ & Positive \\
\hline 5 & 32.1 & Negative & $1 / 640$ & Positive \\
\hline 6 & 284 & Negative & $1 / 1280$ & Positive \\
\hline 7 & 9.05 & Negative & $1 / 160$ & Positive \\
\hline 8 & 26.0 & Negative & $1 / 640$ & Positive \\
\hline 9 & 147 & Negative & $1 / 320$ & Positive \\
\hline 10 & 17.2 & Negative & $1 / 160$ & Positive \\
\hline
\end{tabular}

TPA S/Co: Treponema Pallidum Signal/Cut Off

Table 3. The results of the VDRL and TPHA tests on initial and second visits for the returning donors

\begin{tabular}{lccccc}
\hline \multirow{2}{*}{$\begin{array}{l}\text { Sample } \\
\text { Number }\end{array}$} & \multirow{2}{*}{ Syphilis TPA S/Co } & \multicolumn{2}{c}{ VDRL } & \multicolumn{2}{c}{ TPHA } \\
\cline { 2 - 6 } & & Initial Visit & Second Visit & Initial Visit & Second Visit \\
\hline 2 & 216 & Negative & Negative & $1 / 320$ & $1 / 1280$ \\
\hline 7 & 9.05 & Negative & Negative & $1 / 160$ & $1 / 640$ \\
\hline 9 & 147 & Negative & Negative & $1 / 320$ & $1 / 1280$ \\
\hline
\end{tabular}

TPA S/Co: Treponema Pallidum Signal/Cut Off 
es department of the hospital, were diagnosed as the early phase of sphylitic infection, and were treated medically.

\section{Discussion}

Syphilis remains a public health problem around the world. According to the WHO estimates, there are 12 million new syphilis cases each year, of which approximately $90 \%$ occur in developing countries (1). Over the past three decades, with the increased risk of human immunodeficiency virus (HIV) infection, syphilis has acquired a new potential for morbidity and mortality (1).

WHO recommends that all countries should test blood and blood components for hepatitis B, hepatitis C, HIV and syphilis infections (2). In Turkey, all blood and blood components are tested for these infections.

WHO also cautions that syphilis screening should be undertaken in a highly sensitive manner to minimize the risk of infection via transfusion. Treponemal antibody-specific TPHA or the enzyme immunoassay method are recommended for the screening of syphilis, and for populations with high syphilis incidence, a nontreponemal test, such as VDRL or RPR (2) is usually indicated for screening. In syphilis screening, the traditional methods are nontreponemal RPR or VDRL tests. The samples found to be reactive are then confirmed using treponemal assays; e.g., TPHA and TPPA or fluorescent treponemal antibody absorption (FTA-Abs). However, today, many expert committees and organizations recommend the use of treponemal tests for syphilis screening[1-5]. In this reverse algorithm approach, a reactive treponemal screening test is followed by a quantitative nontreponemal test to diagnose the active disease and monitor the response to treatment. This reverse algorithm consists of a second and different treponemal test used to validate all reactive screening results and identify and resolve nonconforming screening and nontreponemal test results (1, 3-6).

Nontreponemal VDRL and RPR tests produce false negative results in $30 \%$ to $50 \%$ syphilis cases in the primary stage $(1,3-5)$. Studies have shown that the reverse algorithm is highly specific in screening low-risk populations, such as blood donors $(1,4)$. Beginning the screening process with a treponemal test can help detect infection at the same time as or slightly earlier than nonspecific antibody tests (1-4).

Syphilis threatens public health, especially in populations at risk. In many countries, it occurs as a transfusion-transmitted infection. Recent studies in transfusion medicine have focused on the establishment of effective diagnostic algorithms for the screening of blood donors $(1,4)$. There is a clear need for new diagnostic methods and effective diagnostic algorithms for screening (1, 4-6). Many comparative studies on this subject have suggested that there are many advantages of using a reverse algorithm, in which the screening starts with a treponemal test (7-11). The use of treponemal immunoassays for syphilis screening eliminates biologically false positives due to the presence of anticardiolipin antibodies in certain diseases (12-14). In addition, immunoassays can be automated, their results are objective, and they reduce labor costs and increase laboratory efficiency (3, 7-10). However, despite excellent specificity, any test may have a poor predictive value when performed in populations with low disease prevalence, such as blood donors $(1,4)$. Therefore, the reverse algorithm guidelines recommend retesting all samples that are found reactive in a treponemal immunoassay using a nontreponemal test and/or a second, different treponemal test, such as TPPA or TPHA (1, 3-6).

In two different studies reported from Brazil (15, 16), the reverse algorithm involving the use of the CLIA method improved the results of blood bank serologic screening, but still resulted in a 
high rate of false positive results in a healthy population, such as blood donors, which continues to be a problem.

In comparative studies conducted with the CLIA method, it is stated that having high sensitivity and specificity, lower labor costs and faster results, CLIA can be effectively used for screening in laboratories with high sample counts, and it is emphasized that false positive CLIA results can be confirmed by a treponemal test, such as TPHA and TPPA $(7,8,10,11)$.

In the current study, ten samples were found reactive using the CLIA method. Nine of these samples were positive and the remaining one was negative according to the TPHA test. We were not able to repeat the test on the negative sample; therefore, we could not evaluate whether the result was a false positive. Studies show that if the second treponemal test is not reactive, this indicates that the first treponemal test is probably a false screening result and that the donor is less likely to have been exposed to syphilis (17, 18). In this study, the VDRL test was negative for all 10 CLIA-reactive samples, and this result was statistically significant $(\mathrm{p}<0.001)$.

We were able to repeat the tests on new blood samples collected from three prospective donors one month after the initial evaluation. The retested samples were found to have negative VDRL results, but their titers doubled in the TPHA test. These individuals were referred to the infectious diseases clinic for medical evaluation, diagnosis, treatment, and follow-up.

\section{Conclusion}

WHO emphasizes that the sensitivity and specificity of the tests used in donor screening should be high and have a sensitivity limit of at least $99.5 \%$. The sensitivity of nonspecific RPR/ VDRL tests used in syphilis screening is very low compared to specific treponemal tests. In nonspecific tests, false negativity can also be seen especially in early and late syphilis cases. The CLIA method has many advantages, such as high sensitivity and specificity, lower labor costs, and faster results. Of our ten CLIA positive blood donors only three replied to our call which is a limitation of our study. In spite of this, the results of the tests on the initial blood samples revealed high agreement between the two treponemal tests, namely CLIA and TPHA

Of the three blood donors from whom a second blood sample was collected and tested, there were no false positive results for the CLIA and the results were in agreement with the second treponemal test (TPHA) which suggests that CLIA is suitable for syphilis screening. Based on these results, we determined that the use of the CLIA method and reverse algorithm is efficient and sensitive for the syphilis screening of blood donors. Yet, blood centers need to validate this algorithm to evaluate the performance characteristics before replacing it with the present algorithm.

\section{Authors' contribution}

RB - Conceptualization; Data curation; Writing - original draft; Writing -review \& editing

AEK - Data curation; Methodology

\section{Conflict of interest}

There are no conflicts of interest.

\section{References}

1. Kaur G, Kaur P. Syphilis testing in blood donors: an update. Blood Transfusion. 2015;13(2):197. (PMID: 25545876).

2. Organization WH. Screening donated blood for transfusion-transmissible infections: recommendations: World Health Organization; 2010.http://www.who.int/bloodsafety/ScreeningTTI.pdf

3. Loeffelholz MJ, Binnicker MJ. Point-Counterpoint: It is time to use treponemal-specific antibody screening tests for the diagnosis of syphilis. J Clin Microbiol. 2011:JCM. 06347-11. (PMID: 22090405). 
4. Sommese L, De Pascale MR, Capuano M, Napoli C. Efforts in blood safety: Integrated approach for serological diagnosis of syphilis. Asian J Transfus Sci. 2016;10(1):22. (PMID: 27011666). DOI: 10.4103/0973-6247.164267

5. Binder SR, Theel ES. Syphilis testing algorithms: A review. World Journal of Immunology. 2016;6(1):1-8. DOI: $10.5411 /$ wji.v6.i1.1

6. Karaca Y, Çöplü N, Gözalan A, Öncül Ö, Akin L, Esen B. Sifiliz Tanısında Kullanılan Serolojik Testler İçin Akış Şemasının Oluşturulması. Mikrobiyol Bul. 2010;44:35-45. (PMID: 20455397).

7. Li L, Cai B, Tao C, Wang L. Performance evaluation of CLIA for Treponema pallidum specific antibodies detection in comparison with ELISA. J Clin Lab Anal. 2016;30(3):216-22. (PMID: 25716172). DOI: 10.1002/ jcla.21839

8. Wang X, Chen L, Yu Q, Sun A. Evaluation of specific antibodies of blood receiver sera to Treponema pallidum by chemiluminescence immunoassay. Zhonghua shi yan he lin chuang bing du xue za zhi= Zhonghua shiyan he linchuang bingduxue zazhi $=$ Chinese journal of experimental and clinical virology. 2013;27(1):70-2. (PMID: 23855137).

9. Marangoni A, Moroni A, Accardo S, Cevenini R. Laboratory diagnosis of syphilis with automated immunoassays. J Clin Lab Anal. 2009;23(1):1-6. (PMID: 19140205). DOI: $10.1002 /$ jcla.20268

10. Ortiz DA, Loeffelholz MJ. Evaluation of the Lumipulse ${ }^{\circledR}$ G TP-N Chemiluminescent Immunoassay as a Syphilis Screening Test. J Clin Microbiol. 2017:JCM. 00966-17. (PMID: 28878003).

11. Liu L, Xie Y, Dai Z, Zhuo C, Wu Y. Establishment and Evaluation of a One-Step Microplate Chemiluminescence Immunoassay to Detect IgG Antibody Against Treponema Pallidum. J Clin Lab Anal. 2015;29(6):493-
7. (PMID: 25277551). DOI: 10.1002/jcla.21799

12. Tiwari AK, Acharya DP, Dara RC, Arora D, Aggarwal G, Rawat GS. Evaluation of nonspecific treponemal test rapid plasma reagin in comparison with specific treponemal test immunochromatographic assay for screening healthy blood donors. Global J Transfusion Med. 2017;2(1):29. DOI: 10.4103/GJTM.GJTM_46_16

13. Naidu NK, Bharucha Z, Sonawane V, Ahmed I. Comparative study of Treponemal and non-Treponemal test for screening of blood donated at a blood center. Asian J Transfus Sci. 2012;6(1):32. (PMID: 22623840). DOI: 10.4103/0973-6247.95048

14. Wang LN, Li JM. Evaluation of immunoglobulin M and $\mathrm{G}$ Western blot and ELISA for screening antibodies to Treponema pallidum in blood donors. Sex Transm Dis. 2009;36(7):413-6. (PMID: 19387420). DOI: 10.1097/ OLQ.0b013e31819db594

15. Baião A, Kupek E, Petry A. Reverse algorithm for syphilis screening more than halved false positive test results in Brazilian blood donors. Transfus Med. 2014;24(1):64-6. (PMID: 24329733). DOI: 10.1111/ tme. 12095

16. Sandes V, Silva S, Motta I, Velarde L, de Castilho S. Evaluation of positive and false-positive results in syphilis screening of blood donors in Rio de Janeiro, Brazil. Transfus Med. 2017;27(3):200-6. (PMID: 28230309). DOI: $10.1111 /$ tme. 12395

17. Soreng K, Levy R, Fakile Y. Serologic Testing for Syphilis: Benefits and Challenges of a Reverse Algorithm. Clin Microbiol Newsl. 2014;36(24):195. (PMID: 28845073). DOI: 10.1016/j.clinmicnews.2014.12.001

18. Binnicker M. Which algorithm should be used to screen for syphilis? Curr Opin Infect Dis. 2012;25(1):79. (PMID: 22156894). DOI: 10.1097/QCO.0b013e$32834 \mathrm{e} 9 \mathrm{a} 3 \mathrm{c}$ 